\title{
Efectos de Omega-3 en el Sueño: Revisión Sistemática.
}

\section{Effects of Omega 3 on Sleep: Systematic Review.}

\author{
Francisca Toledo ${ }^{1}$, Isabel Sepúlveda ${ }^{1}$, Gustavo Moncada ${ }^{2}$, Constanza Valdés ${ }^{1}$, Raúl Frugone-Zambra ${ }^{1}$
}

1. Desórdenes Temporo mandibulares, Facultad de Odontología, Universidad de Los Andes, Santiago, Chile.

2. Rehabilitación Oral, Facultad de Odontología, Universidad de Los Andes, Santiago, Chile.

* Correspondencia Autor: Gustavo Mondaca |

E-mail: gmoncada@adsl.tie.cl

Trabajo recibido: 29/7/2020

Trabajo revisado: 05/10/2020

Trabajo aprobado: 22/11/2020

\begin{abstract}
RESUMEN
Objetivo: Determinar el efecto de omega-3 sobre la calidad/cantidad de sueño en humanos. Métodos: Se realizó la búsqueda sistemática de artículos (2010-2019), incluyendo pacientes sin limitación de edad/sexo, sometidos a cambio o suplemento de dieta con omega-3 y evaluaciones de sueño. La revisión se realizó bajo los criterios PICOT y PRISMA, la calidad de la evidencia y riego de sesgo fueron evaluados con criterios GRADE. Resultados: Seis ensayos superaron todos los filtros, dos estudios incluyeron pescado en la dieta y cuatro ingesta de cápsulas de omega-3. En cuatro estudios Omega-3 favorece el sueño presentando impacto moderadamente positivo en calidad y alto en cantidad. Los estudios fueron efectuados con variables de alta heterogeneidad, imposibilitando el análisis cuantitativo de los datos. El riesgo de sesgo fue moderado-alto. Conclusión: Omega-3, como suplemento dietético o directamente en los alimentos, interviene como coadyuvante para mejorar el sueño. No se pudo concluir sobre su recomendación de uso clínico en la gestión del sueño debido a la heterogeneidad de las metodologías para medir la intervención, las poblaciones estudiadas y cantidad de ingesta. Se necesitan estudios con mayor estandarización metodológica, para determinar dosis óptima, período de intervención y proporción de ácidos eicosapentaenoico/docosahexaenoico, necesarios para mejorar la calidad y la cantidad del sueño.
\end{abstract}

PALABRAS CLAVE

Omega 3; Ácidos grasos omega 3; Sueño; Trastornos del sueño; Calidad del sueño; Falta de sueño.

Int. J. Inter. Dent Vol. 14(3); 266-270, 2021.

\section{ABSTRACT}

Objective: To determine the effect of omega-3 on the quality/quantity of sleep in humans. Methods: We conducted a systematic search for articles (2010-2019), including patients without age or sex limitation, undergoing omega-3 diet change or supplementation and sleep assessments. The review was conducted under the PICOT and PRISMA criteria, the quality of evidence and risk of bias were evaluated with GRADE criteria. Results: Six trials passed all filters. Two studies included dietary fish and four omega-3 capsule intake. In four studies, omega-3 favored sleep with moderately positive impact on quality and high impact on quantity. The studies were conducted with highly heterogeneous variables, making a quantitative analysis of the data impossible. The risk of bias was moderate to high. Conclusion: Omega- 3 as a dietary supplement or directly in food intervenes as an adjuvant to improve sleep. We could not conclude on its recommendation for clinical use in sleep management due to the heterogeneity of the methodologies to measure the intervention, the populations studied and amount of intake. Studies with greater methodological standardization are needed, to determine optimal dose, intervention period and eicosapentaenoic/docosahexaenoic acid ratio, needed to improve sleep quality and quantity.

\section{KEY WORDS}

Omega 3; Fatty acids omega 3; Sleep; Sleep disorders; Quality of sleep; Poor sleep.

Int. J. Inter. Dent Vol. 14(3); 266-270, 2021.

\section{INTRODUCCIÓN}

El sueño es un requisito biológico básico para la vida participando en conjunto con otras funciones neurobiológicas que regulan numerosos procesos fisiológicos como la recuperación de la fatiga, la reparación de los tejidos, la consolidación de la memoria y la función cerebral, tanto a nivel de la red celular como del sistema nervioso central ${ }^{(1)}$. Las personas total o parcialmente privadas de sueño pueden enfermar rápidamente, ya que al no dormir lo suficiente (3-4 días), generan alteraciones del estado de ánimo, problemas cognitivos, cambios en el sistema inmunológico y somático, además de complicaciones relacionadas con el manejo del dolor ${ }^{(2,3)}$.

Durante una noche de sueño típica, se desarrollan tres a cinco ciclos de fase de sueño no-REM (movimiento ocular no rápido) a REM (movimiento ocular rápido), o ciclo de ritmo ultradiano (en contraste con el ciclo circadiano de 24 horas). Durante el sueño REM o "sueño paradójico", todos los músculos esqueléticos permanecen en estado hipotónico, sin embargo, el SNC y el sistema nervioso autónomo se tornan activos, participando en la reparación tisular ${ }^{(1,3)}$. Durante el sueño se producen series de eventos que permiten la reparación del organismo, 
el restablecimiento energético, la eliminación de radicales libres, la regulación endocrina, la consolidación de la memoria y la respuesta inmune, entre otros ${ }^{(4)}$.

El sueño no reparador se refiere a la sensación de cansancio al despertar y se presenta aproximadamente en el $10 \%$ de la población, siendo queja frecuente de personas con síntomas relacionados con insomnio, fatiga y alteraciones del ánimo ${ }^{(1-3)}$. Diversos estudios muestran directa relación entre sueño alterado y patrones alimenticios no saludables, como dieta baja en frutas, verduras, pescados grasos y cereales integrales, horarios de comidas irregulares, ingesta frecuente y elevada de golosinas ricas en azúcar, grasas saturadas y consumo elevado de bebidas azucaradas ${ }^{(5)}$

Los ácidos grasos poliinsaturados de cadena larga (AGPICL) son componentes nutricionales esenciales para el ser humano, desempeñando procesos fisiológicos fundamentales cumpliendo un rol estructural en los fosfolípidos de las membranas celulares y siendo la base para la síntesis de numerosos mediadores fisiológicos inmunitarios y neurológicos. El ácido linoleico (LA) y el ácido alfa-linolénico (ALA), son los principales AGPICL que dan origen a omega-6 ( $\omega-6)$ y omega-3 $(\omega-3)$ respectivamente ${ }^{(6)}$

Los AGPICL $\omega-3$ son definidos por su doble enlace en el tercer carbono del extremo metilo de la cadena de carbonos y los humanos no disponen de la enzima $\omega-3$ desaturasa necesaria para agregar el doble enlace en el $15^{\circ}$ carbono, no pudiendo sintetizar endógenamente los ALA y AL. Sin embargo el cuerpo humano tiene la capacidad de metabolizar estos ácidos grasos a través de etapas de elongación y desaturación, que en $\omega$-3 (C18:3) es transformado en ácido eicosapentaenoico (EPA, C20:5) y posteriormente en ácido docosahexaenoico (DHA, C22:6,). Los triglicéridos EPA y DHA, se encuentran en animales y vegetales de origen marino, especialmente en pescados con elevado contenido graso como los peces de agua fría (atún, jurel, salmón, anchoa, calamar, otros) y en otras alimentos marinos como mejillón, ostra, camarón ${ }^{(7)}$, adicionalmente se encuentra en alimentos suplementados con $\omega$-3 como leche, huevo, margarinas y otros ${ }^{(8)}$. Y uno de sus roles más importante es el desarrollo neurológico y ocular además de controlar y reducir la inflamación.

Los ácidos grasos $\omega-6$ (C18:2), derivados del AL son alargados en dos carbones (por elongasas y desaturasas) formando el ácido araquidónico (AA, C20:4), encontrándose en dicho estado en carnes de vacuno, cordero y cerdo, en aceites (soya, linaza, canola, oliva y otros.) y frutos secos (almendra, nuez, maní semilla de lino y otros). El AA es un componente estructural de las membranas celulares (fosfolípidos) que es liberado por la fosfolipasa A2 en los primeros estadios de la inflamación y que posteriormente es metabolizado por lipooxigenasas y ciclooxigenasas, para formar eicosanoides, como prostaglandinas, leucotrienos y tromboxanos ${ }^{(9)}$, que juegan un rol en la respuesta inmune, regulación de la coagulación, promoción de la inflamación y contribuyen al equilibrio del perfil lipídico(10)

Dada la información controversial que existe respecto a los efectos de omega-3 en el sueño, la presente revisión sistemática tuvo como objetivo determinar los efectos de su ingesta en la calidad y/o cantidad de sueño en humanos.

\section{METODOLOGÍA}

Protocolo y registro: El presente estudio fue parte del proyecto de investigación 201951 aprobado por el Comité de Ética Científica de la Universidad de Los Andes.

Fuentes de información y búsqueda: Se realizó una búsqueda sistemática en las bases de datos PubMed, Scopus, EMBASE, Google Scholar, Epistemonikos y EBSCO, sin restricción de idioma, publicados entre el 01 de enero del año 2010 al 02 de Junio de 2019, para determinar el efecto de los ácidos grasos omega-3 sobre la calidad y/o cantidad de sueño en pacientes sometidos a cambio de dieta con alimentos ricos en omega 3 o a suplemento con omega 3 . Se utilizaron las palabras claves "omega 3", "omega 3 fatty acids", "fatty acids omega 3", "sleep", "sleep disorders", "quality of sleep" y "poor sleep". No se efectuó búsqueda de literatura gris.

Se aplicaron 4 filtros: título, resumen, duplicados (Zotero ${ }^{\circledR}$ v.5.0) y criterios inclusión - exclusión.

La identificación y selección de los artículos se basó en los criterios de Preferred Reporting Items for Systematic Reviews and Meta-Analyses (PRISMA).

Para definir la calidad de la evidencia y el grado de fuerza de la recomendación de la literatura se utilizaron los criterios Grading of Recommendations Assessment, Development and Evaluation (GRADE).

Los criterios de elegibilidad, búsqueda y recolección de datos se basaron en la estrategia PICOT donde los participantes fueron pacientes sanos y/o con enfermedades o condiciones sistémicas de base; la
Intervención fue el uso de omega-3 en cápsulas o cambio en la dieta incluyendo el consumo de pescado rico en omega-3; el Control fue placebo u otra intervención para mejorar la calidad y/o cantidad de sueño como fármacos, yoga, meditación, u otro, o sin tratamiento; Outcome fue la modificación de la calidad y cantidad de sueño en relación con la intervención y sin limitaciones de Tiempo para el manejo de los resultados.

La pregunta de investigación fue: ¿Es efectiva la ingesta de omega-3 para mejorar la calidad y/o cantidad de sueño, comparado con placebo u otra intervención en seres humanos?

Los criterios de inclusión fueron ensayos clínicos y estudios clínicos aleatorizados controlados, doble o triple ciego, sin restricción de edad, sexo o idioma, con o sin patología de sueño, estudios en humanos sanos y/o con enfermedad o condiciones sistémicas de base o si establecían algún tipo de relación entre omega-3 y sueño.

Proceso de recolección de datos: Los títulos y resúmenes de los artículos fueron identificados, seleccionados y evaluados independientemente por dos investigadores (FT-IS) utilizando un formulario de recolección predeterminado y estandarizado, (Excel ${ }^{\circledR}$ Microsoft 2010), de acuerdo con la estrategia de búsqueda descrita, en casos de desacuerdo recurrieron a la mediación de dos investigadores que actuaron como referís ( $\mathrm{RF}$ y $\mathrm{CV}$ ).

El riesgo de sesgo de las publicaciones, que afecta la validez interna de cada estudio, fue evaluado aplicando los siete criterios de la herramienta GRADE, interpretándose como muy alto riesgo el no cumplimiento de 6-7 criterios, alto riesgo 4-5, moderado 2-3 y bajo riesgo de sesgo el no cumplimiento de 0-1 criterio.

Síntesis y análisis de datos: el resultado principal fue la determinación del impacto de $\omega$-3 en la cantidad o calidad de sueño y como desenlaces secundarios fueron la determinación de niveles plasmáticos de $\omega-3$, frecuencia cardíaca, polisomnografía, actigrafía, niveles de cortisol, uso de manta MMS u otra determinación objetiva. Adicionalmente se analizó la interpretación subjetiva de la calidad/cantidad de sueño según, índice de calidad del sueño de Pittsburgh (PSQI), escala de somnolencia de Epworth (ESS) y/o funcionamiento diurno definido mediante encuestas.

La información extraída se organizó por autor y año, tipo de estudio, característica de los participantes y tamaño de la muestra (experimental y control), intervención, control, resultados, métodos diagnósticos utilizados, métodos de colección de datos, y su metodología de evaluación, conclusiones y limitaciones separados por estudio.

La caracterización de la población estudiada se efectuó por medio de análisis estadístico descriptivo para las variables edad, género y diagnóstico, según el contexto geográfico y sanitario de los participantes.

\section{RESULTADOS}

La búsqueda sistemática identificó 128 estudios por título, de ellos 6 superaron todos los filtros para efectuar la revisión sistemática (Figura 1), todos ellos fueron ensayos clínicos aleatorios (ECA).

Características de los estudios. Un estudio incluyó sólo hombres adultos $^{(11)}$, dos estudios incluyeron sólo mujeres ${ }^{(12,13)}$, uno de ellos mujeres embarazadas y sus recién nacidos ${ }^{(13)}$, dos estudios incluyeron niños entre 4 y 9 años ${ }^{(14,15)}$ y un último estudio incluyó pacientes adultos de ambos $\operatorname{sexos}^{(16)}$. Dos estudios se llevaron a cabo en EEUU y el resto Reino Unido, Noruega, Brasil e Irán. En relación a la presencia de patologías relacionadas, un estudio incluyó sólo pacientes diagnosticados con enfermedad de Parkinson 16, uno con trastorno depresivo mayor tratado con sertralina a dosis terapéuticas 12 y 4 estudios en sujetos sin patologías ${ }^{(11,13-15)}$. Las intervenciones en los grupos experimentales fueron: 2 estudios que modificaron la dieta incluyendo pescado 3 veces por semana ${ }^{(11,14)}$ y los otros 4 suplementaron con cápsulas de omega-3 con $300 \mathrm{mg} / \mathrm{dia}^{(13,16)}, 600 \mathrm{mg} / \mathrm{dia}^{(15)}$ y $1.000 \mathrm{mg} / \mathrm{dia}^{(12)}$. La duración de los estudios fue por períodos continuos de 6 semanas $^{(12)}, 8$ semanas $^{(16)}, 16$ semanas $^{(14,15)}, 20$ semanas $^{(13)}$ y 24 semanas $^{(11)}$. Los grupos controles para los dos grupos con dieta pescado fue dieta cordero, porcino, ave o vacuno, mientras los otros 4 grupos, los controles fueron suplementados con cápsulas placebos (Tabla 1).

Impacto global, medición de las variables y síntesis de datos: $\mathrm{El}$ ácido graso poliinsaturado de cadena larga $\omega-3$, impactó positivamente la variable sueño en 4 estudios ${ }^{(11-13,15)}, 3$ efectuados en adulto ${ }^{(11-13)}$ y uno en niños de 7 a 9 años ${ }^{(15)}$ En dos estudios no se evidenció cambio significativo; uno efectuado en niños (4-6 años) ${ }^{(14)}$ y otro en pacientes enfermos de Parkinson ${ }^{(16)}$. En el estudio de Montgomery ${ }^{(15)}$ el grupo experimental no presentó diferencias significativas con el grupo control, sin embargo, la selección de un subgrupo a cuyos participantes les determinaron los 


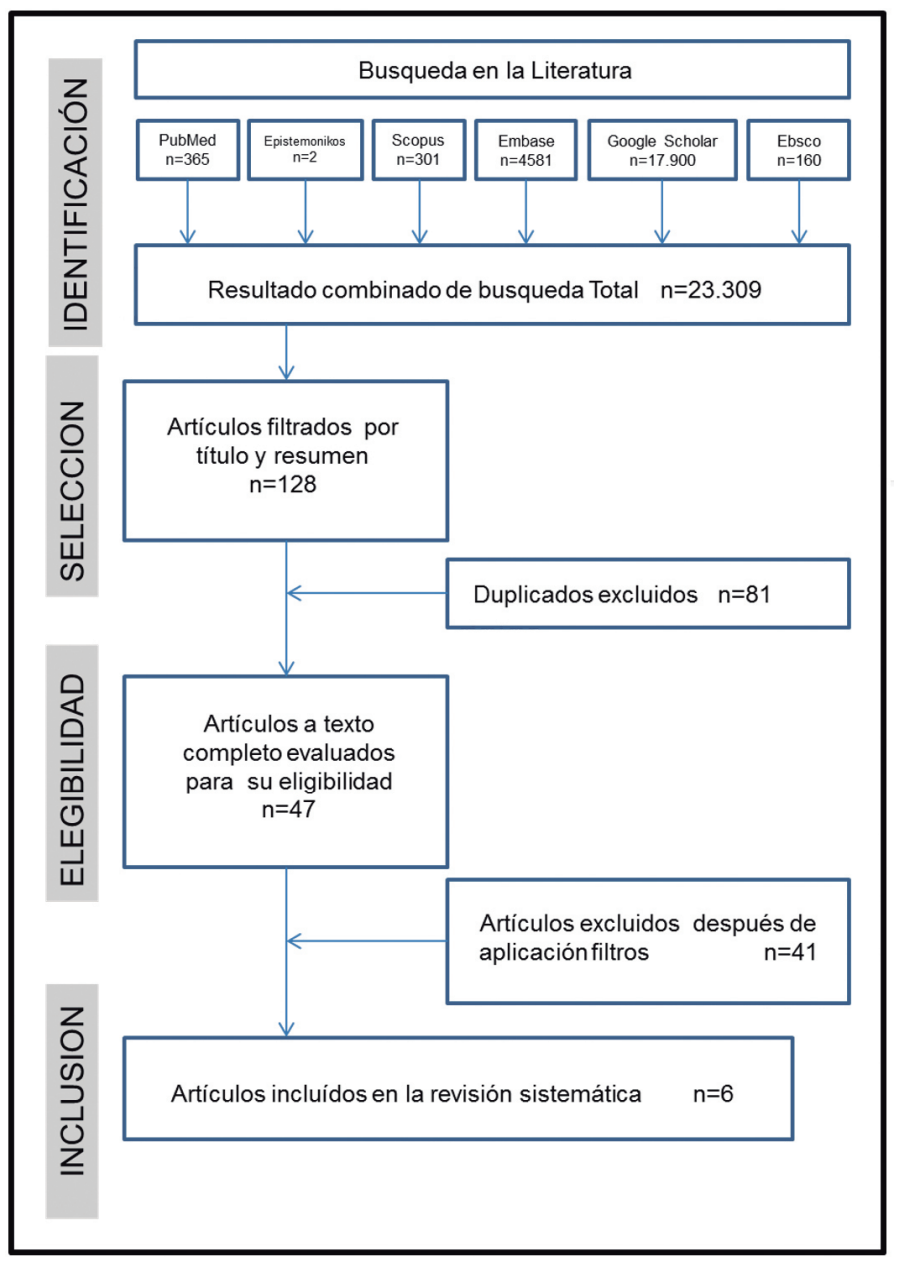

Figura 1. Diagrama de flujo para la selección de los artículos.

Tabla 1: Principales características de los estudios incluidos, separados por autor.

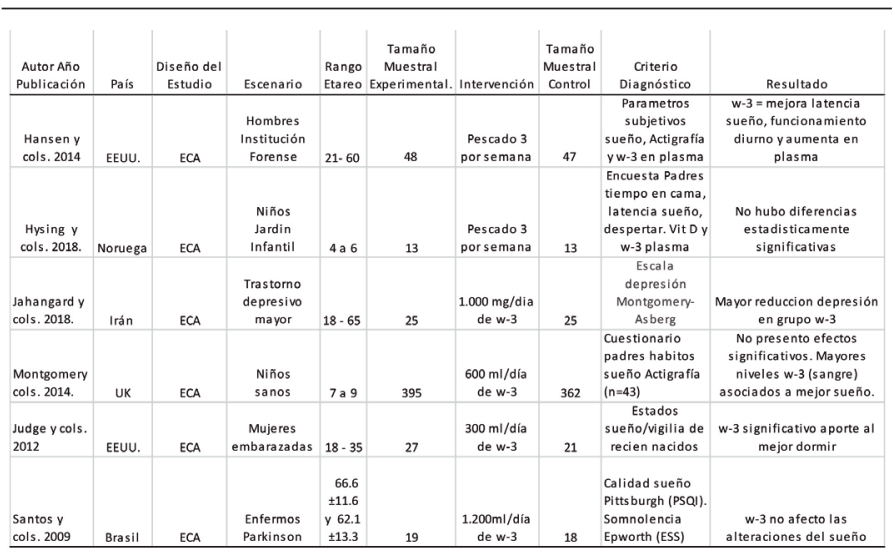

niveles de $\omega-3$ y efectuó actigrafía, permitieron reconocer que la mayor cantidad de $\omega-3$ en plasma se asociaba directamente con mejor calidad de sueño. Ese hecho determinó la inclusión entre los reportes de efecto favorable ${ }^{(15)}$.

De acuerdo a los puntajes obtenidos por el sistema GRADE, un estudio calificó como de alta calidad(15), 4 estudios de moderada calidad ${ }^{(11-14)}$ y uno de baja calidad ${ }^{(16)}$ (Tabla 2 ).

Los riesgos de sesgo según criterios GRADE determinaron que dos estudios fueran clasificados como moderado riesgo de sesgo ${ }^{(13,15)}, 3$ como alto riesgo ${ }^{(11,14,16)}$ y 1 en nivel de no cumplimiento(12) (Tabla 3 ).

Los estudios totalizaron 1.013 pacientes [230 adultos (rango etario 18-78 años) y 783 niños (rango etario 0 a 9 años)], de ellos 527 fueron de los grupos experimentales y 486 placebo o control.
Tabla 2: Análisis de la calidad de los estudios de acuerdo a GRADE.

\begin{tabular}{|c|c|c|c|}
\hline Autor & Titulo & Calidad de la evidencia & Razón \\
\hline $\begin{array}{l}\text { Hansen AL, } \\
2014 .\end{array}$ & $\begin{array}{l}\text { Fish Consumption, Sleep, Daily Functioning, and Heart Rato } \\
\text { Variability. }\end{array}$ & Moderada & $\begin{array}{l}\text { Población no roprosentatativa, bajo n. } \\
\text { Ausencia Heterogenenidad clinica. }\end{array}$ \\
\hline $\begin{array}{l}\text { Hysing, M. } \\
2018 .\end{array}$ & $\begin{array}{l}\text { Fatty fish intake and the efffect on mental health and sleep in } \\
\text { preschool children in FINS-KIDS, a randomized controlled trial. }\end{array}$ & Moderada & $\begin{array}{l}\text { Medición subjetiva del sueño (cuestionario } \\
\text { respondido por padres) }\end{array}$ \\
\hline $\begin{array}{l}\text { Jahangard L, } \\
2018 .\end{array}$ & $\begin{array}{l}\text { Inffuence of adjuvant omega-3-polyunsaturated fatty acids on } \\
\text { depression, sleep, and emotion regulation among oulpatients } \\
\text { with major depressive disorders - Results from a double-blind, } \\
\text { randomized and placebo-controlled clinical trial. }\end{array}$ & Moderada & $\begin{array}{l}\text { Ausencia heterogeneidad muestral } \\
\text { bajo n, medición subjetiva de sueî́o } \\
\text { (cuestionario) }\end{array}$ \\
\hline $\begin{array}{l}\text { Monigomery } \\
\text { P. } 2014 \text {. }\end{array}$ & $\begin{array}{l}\text { Fatty acids and sleep in UK children: subjective and pilot } \\
\text { objective sleep results from the DOLAB study-a randomized } \\
\text { controlled trial. }\end{array}$ & Alta & $\begin{array}{l}\text { Medición objetiva parámettros Omega-3/sueño } \\
\text { (cromatografialactigrafia) Alto n, metodologia } \\
\text { optima }\end{array}$ \\
\hline $\begin{array}{l}\text { Judge MP. } \\
2012 .\end{array}$ & $\begin{array}{l}\text { Maternal consumplicon of a DHA-containing functional food benefits } \\
\text { intant sleep patteming: an early neurodevelopmental measure. }\end{array}$ & Moderada & Bajo n muestral. \\
\hline $\begin{array}{l}\text { Santos, M.N. } \\
\text { 2009. }\end{array}$ & $\begin{array}{l}\text { Double-blind study on the effects of fish oil upon daytime } \\
\text { sleepiness and sleep quality in Parkinson patients. }\end{array}$ & Baja & $\begin{array}{l}\text { n población bejo, nula heterogenecicad olinica, } \\
\text { Medición subjetiva parámetros suefio }\end{array}$ \\
\hline
\end{tabular}

Tabla 3: Riego de sesgo de los estudios, evaluados en los siete criterios GRADE.

\begin{tabular}{lccccccc} 
& $\begin{array}{c}\text { Generacín de } \\
\text { la secuencia }\end{array}$ & $\begin{array}{c}\text { ocultamiento } \\
\text { de la } \\
\text { asignaclón }\end{array}$ & $\begin{array}{c}\text { Cegamiento de } \\
\text { participantesy y } \\
\text { del personal. }\end{array}$ & $\begin{array}{c}\text { cegamiento de } \\
\text { los evaluatores } \\
\text { del resultado. }\end{array}$ & $\begin{array}{c}\text { Datos de } \\
\text { resultado } \\
\text { Incompletos. }\end{array}$ & $\begin{array}{c}\text { Notificación } \\
\text { selectiva ade los } \\
\text { resultados. }\end{array}$ & $\begin{array}{l}\text { Otras fuentes } \\
\text { de sesgo. }\end{array}$ \\
\hline Hansen AL. & Bajo riesgo & Moderado & Bajo riesgo & Alto riesgo & Bajo riesgo & Bajo riesgo & Moderado \\
Hysing M. & Bajo riesgo & Moderado & Bajo riesgo & Alto riesgo & Bajo riesgo & Bajo riesgo & Moderado \\
Jahangard L & Bajo riesgo & Moderado & Moderado & Alto riesgo & Bajo riesgo & Bajo riesgo & Alto riesgo \\
Montgomery P. & Bajo riesgo & Bajo riesgo & Bajo riesgo & Alto riesgo & Bajo riesgo & Bajo riesgo & Moderado \\
Judge MP. & Bajo riesgo & Bajo riesgo & Bajo riesgo & Alto riesgo & Bajo riesgo & Bajo riesgo & Moderado \\
Santos MN. & Bajo riesgo & Moderado & Bajo riesgo & Alto riesgo & Bajo riesgo & Bajo riesgo & Altor riesgo \\
\hline
\end{tabular}

\section{DISCUSIÓN}

La literatura reporta que los aceites de pescados ricos en $\omega-3$ afectan favorablemente la salud humana, presentando moderada a alta evidencia de efecto preventivo de enfermedades cardiovasculares ${ }^{(17)}$, sin embargo, la mas amplia revisión efectuada concluye que presenta reducido o ningún efecto sobre la mortalidad y la salud cardio vascular ${ }^{(18)}$, otros estudios le atribuyen el potencial de alterar la trayectoria de enfermedades como diabetes, cáncer, enfermedad de Alzheimer, demencia, depresión, y salud materno-infantil, incluido el deterioro físico asociado con el envejecimiento(17), especialmente después de conocer el efecto controlador de los patrones inflamatorios de las resolvinas series $E$ y D que actúan reduciendo la activación del inflamosoma ${ }^{(19)}$.

En la presente revisión, en general, se estableció moderada relación entre consumo de $\omega-3$ y calidad de sueño, mientras, se observó alta relación entre el consumo de omega-3 y la cantidad de sueño, con nivel de evidencia moderada y riesgo de sesgo alto a moderado.

En los estudios analizados, la calidad de sueño fue evaluada a través de: cuestionarios de Pittsburg Sleep Quality ${ }^{(16)}$, Epworth Sleepness Scale $(E S S)^{(16)}$, Child Sleep Habits Questionnaire $(\mathrm{CSHQ})^{(15)}$, Strengths and Difficulties Questionnaire $(S D Q)^{(14)}$, Time in Bed $(T I B)^{(14)}$, Sleep Onset Latency (SOL) $)^{(14)}$, Wake after Sleep Onset (WASO)(14), Insomnia Severity Index (ISI)(12) y otros, que impidieron su comparación. Tres de seis estudios concluyeron que existe relación positiva entre el uso de omega-3 y aumento en la calidad del sueño(12,13,15). Estos estudios incluyeron 3 poblaciones diferentes: recién nacidos, niños sanos (7-9 años) y adultos (Media 42 años) con diagnóstico de trastorno depresivo mayor en tratamiento por más de 6 meses. Estos tres grupos no son comparables debido a la diferencia etario. El neonato duerme en promedio $70 \%$ del día y la identificación de los dos tipos de sueño, cuya alternancia regular es común en los mamíferos de todas las especies ${ }^{(20)}$ que incluye "sueño activo o paradojal" (sueño REM) y "sueño quieto u ortodoxo" (sueño no-REM) no son similares al adulto. El sueño REM se caracteriza por baja amplitud y ritmos rápidos en el EEG, con movimientos oculares rápidos y el no-REM, se relaciona con la actividad EEG lenta y de mayor amplitud $^{(21)}$. En los recién nacidos, la mayor parte del tiempo su sueño transcurre solo en REM, el que mantendrá la mayoría de los caracteres electrofisiológicos hasta el fin de la vida. Sin embargo el sueño no-REM se modifica notoriamente a través del tiempo ${ }^{(21)}$. Con posterioridad, se definen las etapas del sueño no-REM hasta que, después del primer año, es posible identificar sus etapas características. Progresivamente, aumenta la proporción del sueño no-REM y disminuye la del REM. Estos fenómenos están estrictamente ligados a la maduración neurológica, a tal punto que, por la proporción de cada tipo de sueño y de etapas no-REM puede aproximarse la edad cronológica de los sujetos en la polisomnografía ${ }^{(20)}$. En los años sucesivos, la cantidad de sueño REM se estabiliza en alrededor del $20 \%$ del total del sueño, sin embargo, sobre 
los 50-60 años las etapas más profundas del sueño no-REM reducen su presencia, dominando las etapas más superficiales con un valor constante o levemente disminuido del sueño $\mathrm{REM}^{(21)}$. Por tanto, no es posible homologar los resultados de cada estudio, dado que la arquitectura del sueño es diferente en cada etapa de la vida y adicionalmente los cambios de hábitos, rutinas, carga laboral y siestas, contribuyen a diferencias en la calidad y cantidad de sueño en cada etapa de la vida(21).

En relación al tipo de intervención y calidad de sueño, los estudios no presentaron diferencia significativa entre el consumo de pescado y/o la ingesta de omega-3 aumentando ambos la calidad del sueño ${ }^{(11,14,16)}$ a pesar de las diferencia muestral que incluyeron niños sanos (4-6 años), hombres adultos (media 42 años) y pacientes con enfermedad de Parkinson (>60 años) que presentan trastorno de sueño entre $74 \%$ $98 \%{ }^{(22)}$ y además asociados al compromiso cognitivo, uso de fármacos, cambios bioquímicos propios de la enfermedad, la edad, el uso de fármacos antiparkinsonianos como levodopa que incluye otros trastornos como disquinesias, mioclonías fragmentarias nocturnas, somnolencia y alucinaciones, que también alteran el dormir(21).

Adicionalmente, en los estudios realizados en niños, los cuestionarios fueron contestados por los padres o tutores, aumentando la posibilidad de sesgo, imposibilitando objetivar los resultados. Estudios previos han demostrado ligera sobrestimación de la calidad del sueño cuando los padres son consultados ${ }^{(23)}$

De los tres estudios que mostraron mejora significativa en la calidad de sueño, dos se realizaron en población sana ${ }^{(13,15)}$; en cambio un estudio12 incluyó población con depresión mayor, que fue manejada con vitamina $\mathrm{D}$, higiene de sueño, aumento de la actividad física y además suplemento de omega-3, donde el conjunto de dichas variables podrían haber contribuido a modificar positivamente la calidad de sueño. Por otra parte 3 estudios $^{(11,14,16)}$ no mostraron diferencias significativas en la calidad de sueño, lo que podría deberse al uso de cuestionarios no estandarizados y presencia de patologías complejas de los pacientes, como el Parkinson ${ }^{(16)}$

Con respecto a la medición objetiva de la cantidad de sueño, dos estudios $^{(11,15)}$ utilizaron la actigrafía, registrando los movimientos de la mano, mediante un sensor colocado en la muñeca del paciente que transmite los datos a un computador para analizar los períodos sueño/ vigilia $^{(24)}$.Otro estudio ${ }^{(13)}$ midió objetivamente la cantidad de sueño de los recién nacidos por medio de "MMS", manta que percibe el movimiento del cuerpo, determinando el sueño activo y los despertares, que junto a las mediciones de los nivel de $\omega$-3 en sangre del recién nacido y de su madre, observa que los bebés de las madres que habían consumido $\omega-3(300 \mathrm{mg}$ DHA) en forma de barras de cereal, presentaban significativamente menos despertares en el sueño activo y pasivo en comparación con el grupo placebo ${ }^{(13)}$. Esto podría explicarse porque el DHA suplementado durante el embarazo podría proporcionar ventajas en el desarrollo de SNC del bebé inmediatamente después del nacimiento, lo que también podría relacionarse con el rendimiento cognitivo posterior ${ }^{(13)}$.

Para los tres artículos se determinó la cantidad de sueño, duración, episodios de despertares y latencia y a todos los pacientes se les realizó el registro pre y post intervención de los niveles sanguíneos de $\omega$-3 (600 mg a 1.000 mg día) y/o pescado, (150 a 300grs. de salmón, 3 veces a la semana. En el estudio realizado por Montgomery y cols. ${ }^{(15)}$ en niños de 7-9 años, utilizando suplemento de $600 \mathrm{mg}$ de $\omega$-3, la duración total del sueño aumentó 58 minutos y hubo significativa reducción de 7 despertares nocturnos menos por noche y la latencia de sueño no presentó diferencias significativas entre ambos grupos. Además el grupo $\omega$-3 reveló mejoría en la eficiencia del sueño, respecto del control. Estos datos coinciden con los encontrados en la población adulta del estudio de Hansen cols. ${ }^{(11)}$, intervenidos con el consumo de salmón 3 veces a la semana (150-300grs.) presentando disminución significativa en el tiempo real de sueño del grupo control (con dieta de 150 a $300 \mathrm{grs}$ de pollo o carne,) y aumento en la latencia de sueño del mismo grupo en comparación con el grupo intervención ${ }^{(11)}$

A pesar de la débil evidencia, sobre la ingesta de omega 3 para mejorar las condiciones del sueño, es importante valorar los conocimientos acerca de la nutrición y las respuestas fisiológicas normales en los seres humanos. La dieta occidental actual presenta una proporción de ácidos grasos $\omega-6: \omega-3$ de aproximadamente 15:1, que comparada con la dieta paleolítica en la que los seres humanos evolucionaron, y vivieron durante la mayor parte de su existencia, tenía proporción 1:1 y además era alta en antioxidantes ${ }^{(7)}$ Los cambios en los hábitos alimenticios, los alimentos naturales versus procesados y el desarrollo agrícola en los últimos años causaron severos cambios en la relación $\omega-6: \omega-3$. La disminución del consumo de alimentos enriquecidos con DHA y el aumento del consumo de aceites vegetales enriquecidos con $\omega-6$ son los responsable de la relación 15:1. La dieta occidental actual promueve la patogénesis de muchas enfermedades crónicas como cardiovasculares, inflamatorias, autoinmunes y neurodegenerativas, mientras que la dieta enriquecida en $\omega-3$ ejerce efectos cardio-protectores, inmunosupresores y neuroprotectores ${ }^{(25)}$. La relación ácido araquidónico $(\mathrm{AA})$ :DHA más baja suprime los trastornos mencionados anteriormente. La American Heart Association recomienda la ingesta de pescado dos veces por semana y que las personas con enfermedad coronaria consuman 1gr/día de EPA+DHA de pescado azul o suplementos, destacando que las mejores fuentes de $\omega$ - 3 en la dieta son el pescado o el aceite de pescado como suplemento. Es importante considerar que la ingesta de $\omega-3$ presenta efectos antiinflamatorios y reguladores sobre el eje hipotalámico-pituitarioadrenal (HPA). El eje HPA es parte importante del sistema de respuesta al estrés y también juega rol crítico en el sueño. Una de las funciones importantes del eje HPA es mantener el estado de alerta y regular el sueño. Cuando el eje HPA está desregulado, puede provocar interrupción del sueño y su hiperactividad puede afectar varias etapas del sueño. La inflamación crónica en el cuerpo sobre-activa el eje HPA pudiendo alterar la calidad y cantidad del sueño. Al introducir los ácidos grasos $\omega-3$, la inflamación puede disminuir ${ }^{(26)}$ y controlando la inflamación, las citocinas proinflamatorias reducen su circulación. Tales citocinas de inflamación, también activan la respuesta al estrés en el eje HPA, que puede promover la interrupción del sueño. Existen múltiples evidencias que sugieren que el aumento de la inflamación, especialmente la inflamación crónica, se asocia con dificultades para dormir ${ }^{(27)}$. El eje HPA, estructuralmente compuesto por el hipotálamo, glándula pituitaria y glándula suprarrenal, donde el hipotálamo envía señales a la glándula pituitaria por medio de la neurohormona, la hormona liberadora de corticotrofina $(\mathrm{CRH})$, induce a que la glándula pituitaria envíe señales a la glándula suprarrenal a través de la neurohormona adrenocorticotropina (ACTH). Cuando un individuo está expuesto a un factor estresante, el hipotálamo produce $\mathrm{CRH}$, que luego desencadena la liberación de $\mathrm{ACTH}$, la que se dirige a diversos sistemas. La ubicación objetivo de importancia para el sueño es la glándula suprarrenal, que desencadena la liberación de cortisol. La hormona liberadora de corticotropina $(\mathrm{CRH})$, regulada por el hipotálamo, hace que el eje HPA incremente su actividad, asociando el aumento de $\mathrm{CRH}$ circulante al aumento de la vigilia(28) y en un estudio de hombres adultos sanos sin trastornos del sueño, la CRH disminuyó el sueño de ondas lentas al tiempo que aumentó el sueño ligero y la vigilia(29). La $\mathrm{CRH}$ también se puede producir en respuesta al estrés, este hecho inicia un ciclo de cambios que resultan en problemas crónicos de sueño. A medida que la CRH excesiva sobre activa el eje HPA, se perturba el sueño y reducirá la capacidad para regular la respuesta futura al estrés, hecho que se asocia con el eje HPA funcionando constantemente en hiperactividad. La actividad de ACTH también es de particular interés, por la activación de la glándula suprarrenal por ACTH desencadenando la liberación de hormonas de estrés glucocorticoides El glucocorticoide más relevante para la función del eje HPA y el sueño es el cortisol. En una respuesta saludable del eje HPA, el cortisol es la neurohormona crítica para la desactivación de la respuesta al estrés del eje HPA, cuya hiperactivación se ha relacionado con depresión, dificultades para dormir y ansiedad hechos que demuestran los beneficios de la suplementación con ácidos grasos $\omega$-3 sobre el sueño.

En los estudios sobre madres e hijos se observó que el aumento del consumo de $\omega-3$ y/o pescado condujo a incrementos en los niveles séricos de DHA y EPA, sin embargo, no concluyeron necesariamente que $\omega$-3 mejora la calidad-cantidad de sueño probablemente por la alta demanda anabólica de DHA en el embarazo. Si bien es cierto que la ingesta de $300 \mathrm{mg} /$ día de DHA no modifica las medidas bioquímicas del lactante o la madre, el hecho de ingerir 500 a $1000 \mathrm{mg} /$ día aumenta las concentraciones de DHA. Esto, en niños sanos, demostrándose también que el mayor porcentaje de DHA en la sangre se asocia con la mejor calidad de sueño(12) Por último, en estudios realizados sobre consumo de pescado en adultos durante seis meses mejoran la latencia del sueño. Por lo tanto, existiría relación dosis-respuesta entre la ingesta de DHA en la dieta materna durante el embarazo y las medidas bioquímicas de la madre y el lactante.

A pesar que todos los estudios primarios fueron prospectivos, diseñados como ensayo clínico aleatorizado, la evaluación de sesgo detectó un conjunto de dificultades, dado que posterior a la aleatorización, el ocultamiento de las asignaciones al grupo control o intervención no fue logrado por todos los estudios, hecho que impidió que los involucrados en el ensayo perdieran la imparcialidad de su participación. Adicionalmente el cegamiento o enmascaramiento también presentó dificultades importantes, por ejemplo dada la dificultad para cegar el olor o consistencia del pescado en la dieta. Todos los estudios mostraron ausencia de recomendaciones a los pacientes para modificar sus dietas, que hubiera permitido alcanzar el objetivo de incorporar $\omega-3$ reduciendo la competencia con otras grasas de la dieta.

Finalmente, a pesar que todos los estudios fueron ensayos clínicos 
aleatorios, la alta heterogeneidad de los grupos poblacionales estudiados y la diversidad de las variables para evaluar la intervención, definidas por cada estudio, impidieron realizar el análisis cuantitativo de los datos (meta-análisis), especialmente porque los estudios fueron efectuados en poblaciones de muy diferente edad, sexo, cantidad de sujetos, método de evaluación y cantidad de incorporación del suplemento $\omega$-3. La mayoría de los estudios efectuó las observaciones a través de encuestas o en el caso de los niños, respondidas por los padres. No siendo generalizada la realización de mediciones de biomarcadores detectables en saliva, sangre u otra medición objetiva común para definir los efectos de la intervención.

\section{CONCLUSIONES}

Se observa moderado efecto en el uso de omega 3 como coadyuvante para mejorar la cantidad y calidad de sueño, ya sea ingerido como suplemento o directamente en los alimentos. Los estudios presentaron gran heterogeneidad tanto en las metodologías para medir la intervención calidad/cantidad de sueño, en las poblaciones estudiadas como en la forma de ingesta de omega-3, imposibilitando concluir recomendaciones de uso clínico. Son necesarios estudios con mayor rigurosidad metodológica, para determinar indicación, efectos de mayor validez, como dosis óptima, período de intervención y proporción EPA/DHA que mejore la calidad y cantidad de sueño.

\section{FINANCIAMIENTO}

Ninguno

\section{CONFLICTO DE INTERÉS}

Los autores declaran no presentar conflictos de interés

\section{Bibliografía}

1. Watson NF, Badr MS, Belenky G, Bliwise DL, Buxton OM, Buysse D, et al Recommended amount of sleep for a healthy adult: a joint consensus statement of the American Academy of Sleep Medicine and Sleep Research Society. Sleep. 2015;38:843-4.

2. Stone CR, Haig TR, Fiest KM, McNeil J, Brenner DR, Friedenreich CM. The association between sleep duration and cancer-specific mortality: a systematic review and meta-analysis. Cancer Causes Control. 2019;30:501-25.

3. García-Perdomo HA, Zapata-Copete J, Rojas-Cerón CA. Sleep duration and risk of all-cause mortality: a systematic review and meta-analysis. Epidemiol Psychiatr Sci. 2019;28:578-88.

4. Watson NF, Badr MS, Belenky G, Bliwise DL, Buxton OM, Buysse D, et al Joint consensus statement of the American Academy of Sleep Medicine and Sleep Research Society on the recommended amount of sleep for a healthy adult: methodology and discussion. Sleep. 2015;38:1161-83.

5. Godos J, Ferri R, Caraci F, Cosentino FI, Castellano S, Galvano F, et al Adherence to the mediterranean diet is associated with better sleep quality in italian adults. Nutrients. 2019;11:(5):976.

6. Burr GO, Burr MM. On the nature and role of fatty acids essential in nutrition. $J$ Biol Chem. 1930;86:587-621.

7. Simopoulos AP. Genetic variants in the metabolism of omega- 6 and omega- 3 fatty acids: their role in the determination of nutritional requirements and chronic disease risk. Exp Biol Med. (Maywood) 2010;235:785-95

8. Lupton JR, Blumberg JB, L'Abbe M, LeDoux M, Rice HB, von Schacky C, et al. Nutrient reference value: non-communicable disease endpoints--a conference report. Eur J Nutr. 2016;55:S1-10.

9. Holinstat M, Boutaud O, Apopa PL, Vesci J, Bala M, Oates JA, et al. Proteaseactivated receptor signaling in platelets activates cytosolic phospholipase a2a differently for cyclooxygenase-1 and 12-lipoxygenase catalysis. Arterioscler Thromb Vasc Biol. 2011:31:435-42.

10. Bagga D, Wang L, Farias-Eisner R, Glaspy JA, Reddy ST. Differential effects of prostaglandin derived from omega- 6 and omega-3 polyunsaturated fatty acids on COX-2 expression and IL-6 secretion. Natl Acad Sci. U S A. 2003:100:1751-6.

11. Hansen AL, Dahl L, Olson G, Thornton D, Graff IE, Frøyland L, et al. Fish consumption, sleep, daily functioning, and heart rate variability. J Clin Sleep Med. 2014;10:567-75

12. Jahangard L, Sadeghi A, Ahmadpanah M, Holsboer-Trachsler E, Bahmani DS Haghighi M, et al. Influence of adjuvant omega-3-polyunsaturated fatty acids on depression, sleep, and emotion regulation among outpatients with major depressive disorders-Results from a double-blind, randomized and placebo-controlled clinical trial. J Psychiatric Res. 2018;107:48-56.

13. Judge MP, Cong $X$, Harel $O$, Courville AB, Lammi-Keefe CJ. Maternal consumption of a DHA-containing functional food benefits infant sleep patterning: an early neurodevelopmental measure. Early Human Development. 2012;88:531-7. 14. Hysing M, Kvestad I, Kjellevold M, Kolden Midtbø L, Graff I, Lie $\varnothing$, et al. Fatty Fish intake and the effect on mental health and sleep in preschool children in finskids, a randomized controlled trial. Nutrients. 2018;10:1478.

15. Montgomery P, Burton JR, Sewell RP, Spreckelsen TF, Richardson AJ. Fatty acids and sleep in UK children: subjective and pilot objective sleep results from the DOLAB study-a randomized controlled trial. J Sleep Res. 2014;23:364-88.

16. Santos MN, Mateus CE, Ferraz AC, Louzada FM. Double-blind study on the effects of fish oil upon daytime sleepiness and sleep quality in parkinson patients. Sleep Sci. 2019;2:36-9.

17. Jeromson S, Gallagher IJ, Galloway SD, Hamilton DL. Omega-3 fatty acids and skeletal muscle health. Mar Drugs. 2015;13:6977-7004.

18. Abdelhamid AS, Brown TJ, Brainard JS, Biswas P, Thorpe GC, Moore HJ, et al Omega-3 fatty acids for the primary and secondary prevention of cardiovascular disease. Cochrane Database Syst Rev. 2018;7:CD003177.

19. Bäck M, Yurdagul Jr A, Tabas I, Öörni K, Kovanen PT. Inflammation and Its resolution in atherosclerosis: mediators and therapeutic opportunities. Nat Rev Cardiol. 2019;16:389-406.

20. Ellingson RJ. Development of wakefulness-sleep cycles and associated EEG patterns in mammals. Cap 9. In: Clemente CD, Purpura DP, Mayer FE, Ed. Sleep and the maturing nervous. Academic Press Inc., New York;1972; p.165-174.

21. David P, Blanco M, Pedemonte M, Velluti R, Tufik S. Medicina del sueño. 1a. ed. Mediterráneo: Stgo, Chile, 2008.

22. Diekelmann S, Born J. The memory function of sleep. Nat Rev Neurosci. 2010;11:114-26.

23. Sekine M, Chen X, Hamanishi S, Wang H, Yamagami T, Kagamimori S. The validity of sleeping hours of healthy young children as reported by their parents. J. Epidemiol. 2002;12:237-42.

24. Kanady JC, Drummond SP, Mednick SC. Actigraphic assessment of a polysomnographic-recorded nap: A validation study. J Sleep Res. 2011;20:214-22. 25. White B. Dietary fatty acids. Am Fam Physician. 2009;80:345-50.

26. Motivala SJ, Sarfatti A, Olmos L, Irwin MR. Inflammatory markers and sleep disturbance in major depression. Psychosom Med. 2005;67:187-94.

27. Song C, Lin A, Bonaccorso S, Heide C, Verkerk R, Kenis G, et al. The inflammatory response system and the availability of plasma tryptophan in patients with primary sleep disorders and major depression. J Affect Disord 1998:49:211-9. 28. Buckley TM, Schatzberg AF. On the interactions of the hypothalamic-pituitaryadrenal (HPA) axis and sleep: normal HPA axis activity and circadian rhythm exemplary sleep disorders. J Clin Endocrinol Metab 2005;90:3106-14.

29. Holsboer F, Von Bardeleben U, Steiger A. Effects of intravenous corticotropinreleasing hormone upon sleep-related growth hormone surge and sleep EEG in man. Neuroendocrinology. 1988;48:32-8. 